\title{
Changes in Inpatient Staffing Following Implementation of New Residency Work Hours
}

\author{
Jennifer M. Oshimura, MD, Jeffrey Sperring, MD¹, Benjamin D. Bauer, MD¹, Aaron E. Carroll, MD, MS ${ }^{1}$, \\ Daniel A. Rauch, MD, FAAP, FHM²
}

${ }^{1}$ Department of Pediatrics, Indiana University School of Medicine, Riley Hospital for Children, Indianapolis, Indiana; ${ }^{2}$ Department of Pediatrics, Mount Sinai School of Medicine, Elmhurst Hospital Center, Elmhurst, New York.

BACKGROUND: In 2011, the Accreditation Council for Graduate Medical Education added additional resident work-hour restrictions that limited the number of hours residents could work, with increased emphasis on attending supervision.

OBJECTIVE: Our objective was to determine how residency programs have responded to residency work hours, specifically assessing residency night float systems and in-house attending physicians.

DESIGN: In May 2012, an electronic survey was sent to all US pediatric residency training programs via the Association of Pediatric Program Directors listserv with e-mail reminders to nonresponding programs. We analyzed data to assess the use of resident night float systems, admission caps, and attending physicians in-house at night.

RESULTS: Out of 198 programs contacted, 152 programs responded (77\% response rate). Residency programs utilizing a night float system increased from $43 \%$ to $71 \%$ after new work hours were implemented. Overall use of resident admission caps did not change significantly. Twenty-three percent of programs increased the number of attending physicians in-house at night; $57 \%$ of those programs increased the number of pediatric hospitalist attendings, whereas $37 \%$ increased the number of pediatric intensivists. There is a trend toward increased pediatric hospitalist attending in-house 24/7 coverage. Of programs without $24 / 7$ coverage, $26 \%$ plan to add coverage within 5 years. Only $12 \%$ of programs have no in-house attending coverage at night.

CONCLUSIONS: Although programs vary in their response to changes in residency work restrictions, they most commonly utilize night float systems and increased the amount of in-house attending coverage at night, especially pediatric hospitalist attendings. Many programs plan to add 24/7 pediatric hospitalist coverage within 5 years. Journal of Hospital Medicine 2014;9:640-645. (C) 2014 Society of Hospital Medicine
Long work hours with abnormal schedules and extended on-call periods are common for physicians. Prior to resident work-hour restrictions, studies showed that sleep-deprived residents were at increased risk for making errors with decreased decision-making abilities. $^{1,2}$ Resident work-hour restrictions and increased attending supervision regulations were initially implemented in 1989 in New York due to concerns for patient safety. ${ }^{3}$ In 2003, the Accreditation Council for Graduate Medical Education (ACGME) adopted this 80-hour work week standard nationally and restricted residents to a maximum of 30 hours of continuous clinical responsibilities. ${ }^{4}$ Due to concern that residents working extending periods of time were at risk for making serious medical errors, ${ }^{5-7}$ the ACGME mandated additional resident work-hour restrictions in July 2011. ${ }^{8}$ These changes reduced the maximum hours of continuous clinical responsibilities

*Address for correspondence and reprint requests: Jennifer M. Oshimura, MD, Department of Pediatrics, 705 Riley Hospital Drive, Indianapolis, IN 46202; Telephone: 414-232-3034; Fax: 317-948-2959; E-mail: joshimur@iupui.edu

Additional Supporting Information may be found in the online version of this article.

Received: April 29, 2014; Revised: July 3, 2014; Accepted: July 8, 2014 2014 Society of Hospital Medicine DOI 10.1002/jhm.2242

Published online in Wiley Online Library (Wileyonlinelibrary.com). from 30 to 16 hours for interns, and 28 hours for upper-level residents, including 4 hours for transition of patient care. Continuous on-site supervision by attending physicians is not mandated, but programs had to accommodate for the increased emphasis on attending services and supervision of residents, especially at night. ${ }^{6,8}$ Our previous study in 2010, prior to the implementation of new resident work hours, showed $84 \%$ of pediatric residency programs had pediatric hospitalists. Of those, $24 \%$ had $24 / 7$ pediatric hospitalist coverage, $22 \%$ of pediatric residency programs had no in-house attendings at night, and $31 \%$ of programs at that time planned on adding $24 / 7$ pediatric hospitalist coverage within the next 5 years if further resident work-hour restrictions were implemented. ${ }^{9}$

The objective of this study was to determine how inpatient staffing of teaching services within pediatric residency programs has changed following this recent transition of additional resident work hours. We also sought to define current attending physician staffing and explore attending physicians' overnight responsibilities following new ACGME standards, specifically looking at the role of pediatric hospitalists.

\section{METHODS}

We developed a Web-based electronic survey consisting of 23 questions. Many of these questions were 
multiple choice or numerical values with the option to comment. The survey gathered data on the demographics of pediatric residency programs including: the number of residents in each program, patient admission caps (the total number of patients the resident team can admit overnight), and the use of resident night floats (a resident team working the overnight shift, admitting and cross-covering patients who will be handed over to a day team in the morning).

We also examined the number of pediatric providers at night, the use of pediatric hospitalists, and specifically the use of attendings in-house at night and their overnight responsibilities.

The survey was first pilot tested by pediatric hospitalists for face validity. It was reviewed and approved by the Association of Pediatric Program Directors (APPD) research task force. The survey was sent to 198 US pediatric residency programs via the APPD listserve in May 2012. Program directors were given the option of completing it themselves or designating someone else to complete it. We sent 2 e-mail reminders via the listserve with individual e-mail reminders to nonresponding programs. We sent follow-up e-mails and phone calls to programs with current night float systems to clarify their use of resident night float prior to implementation of new workhour restrictions. Duplicate responses from a program were removed by initially removing the 1 with incomplete data. If both responses were complete, we removed the second response. We analyzed the use of

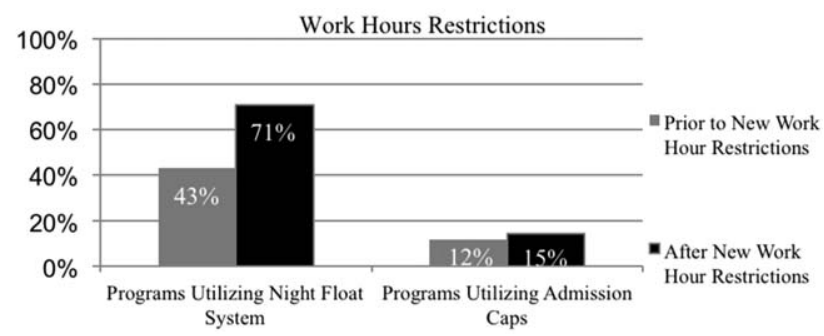

FIG. 1. Changes to night float and admission caps following the new workhour restrictions. Change in night float is statistically significant $(P<0.0001)$. Change in admission caps is not statistically significant $(P=0.52)$. resident night float systems and admission caps, as well as the use of attending physicians in-house at night, using a $z$-score and $\chi^{2}$ test.

The institutional review board of the Indiana University School of Medicine reviewed this study.

\section{RESULTS}

Out of 198 pediatric ACGME programs contacted, 152 responses were received, which is a $77 \%$ response rate. This represented 7828 pediatric residents, or $79 \%$ of total US pediatric residents. Average program size was 52 residents (range, 6-168 residents; median, 41). This average program size was similar to the ACGME average program size of 50 residents. Sorting our response rate by program size, all 58 large ACGME programs responded (programs with over 50 residents). Eighty-four percent $(57$ programs $)$ of medium-sized programs responded (programs with 30-50 residents). Fifty-one percent (37 programs) of small programs responded (programs with $<30$ residents).

\section{Changes in Resident Staffing}

Residency programs utilizing night float systems increased from $43 \%$ before to $71 \%$ after new work hours were implemented $(P<0.0001)$. Overall use of resident admission caps did not significantly change $(12 \%-14.5 \%, P=0.52)$ (Figure 1).

\section{Changes in Attending Physicians In-House at Night}

Following implementation of new resident work-hour restrictions, $23 \%$ of programs increased the number of attending physicians in-house at night. Of these programs, $57 \%$ (20 programs) increased the number of pediatric hospitalist attendings in-house at night, whereas $37 \%$ increased the number of pediatric intensive care unit attendings (Figure 2). When asked the reason for increased attending physician presence in-house, $71 \%$ of programs attributed this change to increased resident work-hour restrictions, and 37\% attributed it to increased patient census. Other common reasons cited included increased patient acuity as well as improved resident supervision and education.

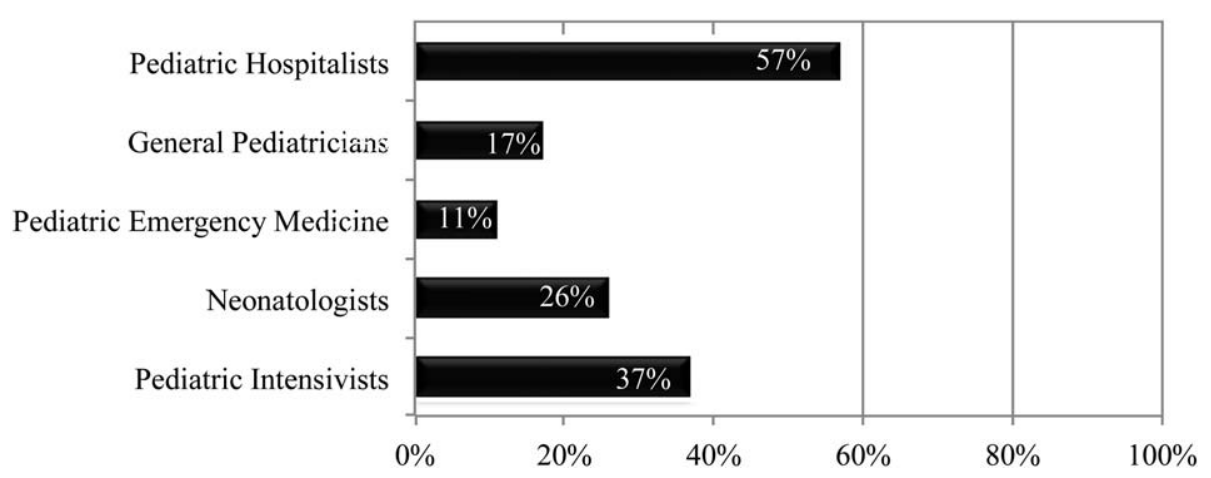

FIG. 2. If the number of attending physicians in-house at night has increased, what specialties have these in-house attending physicians come from ( $\mathrm{n}=35$ programs)? 


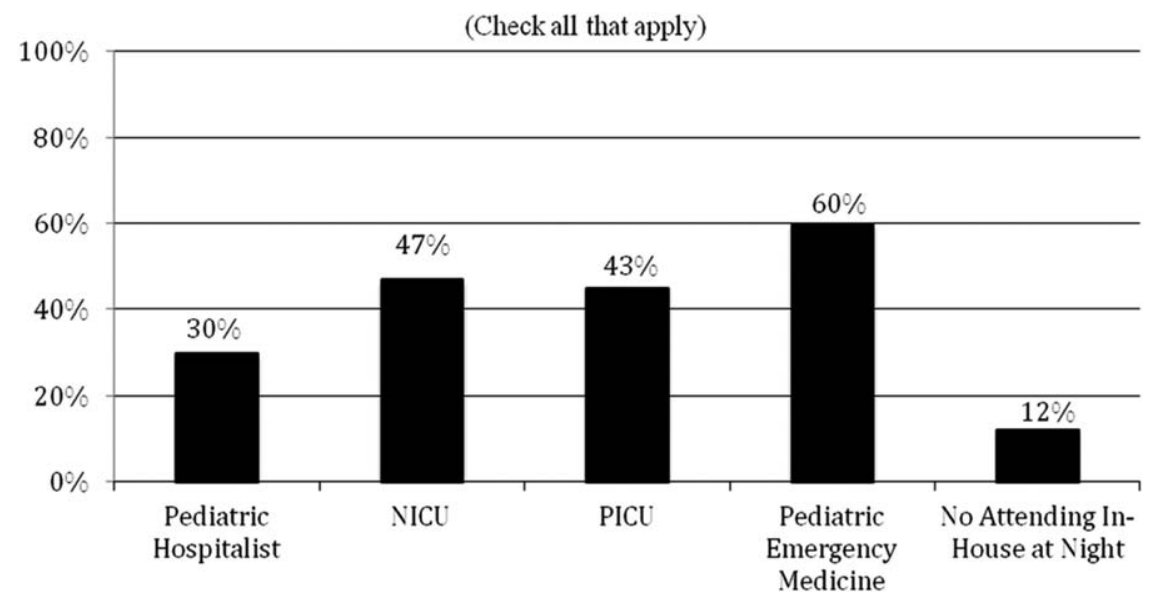

FIG. 3. Do you have attendings in-house at night? (Check all that apply.) This includes both partial nighttime coverage (for example, until midnight) as well as overnight coverage. Abbreviations: NICU, neonatal intensive care unit; PICU, pediatric intensive care unit.

Currently, $30 \%$ of responding programs have pediatric hospitalists in-house at night. This nighttime inhouse coverage includes both partial nighttime coverage (for example, until midnight) and overnight coverage. Forty-seven percent have neonatal intensive care unit (NICU) and $43 \%$ have pediatric intensive care unit (PICU) in-house attending coverage. Sixty percent of responding programs have pediatric emergency medicine attendings in-house at night. Only $12 \%$ of programs have no in-house attending night coverage at all (Figure 3).

Although there was a trend toward increased pediatric hospitalist attendings in-house 24/7, this did not meet statistical significance $(16 \%-20 \%, P=0.36)$. Programs with night hospitalist coverage were more likely to be small $(<30$ residents $)$ or large $(50+$ residents), compared to medium-sized programs (30-49 residents) $(P<0.0032)$. Thirty-eight percent of small programs, $14 \%$ of medium programs, and $41 \%$ of large programs have in-house night hospitalist coverage.

All large programs have some attending physicians in-house at night (NICU, PICU, pediatric emergency medicine, or hospitalist). All programs with no attendings in-house at night have fewer than 46 residents. Of programs with pediatric hospitalists (119), hospitalist attendings have in-house daytime-only coverage in approximately half the responding programs $(48 \%)$. The other half of the programs is split between providing some evening coverage $(22 \%)$ and $24 / 7$ coverage $(26 \%)$ (Figure 4$)$.

\section{Responsibilities of In-House Pediatric Hospitalist Attendings at Night}

Hospitalist attendings who are in-house at night have a variety of night responsibilities including approving admission and transfers (65\%), teaching residents $(74 \%)$, consulting for other services $(65 \%)$, and consulting for residents $(65 \%)$. They vary in how they staff new patient admissions, with $65 \%$ of programs

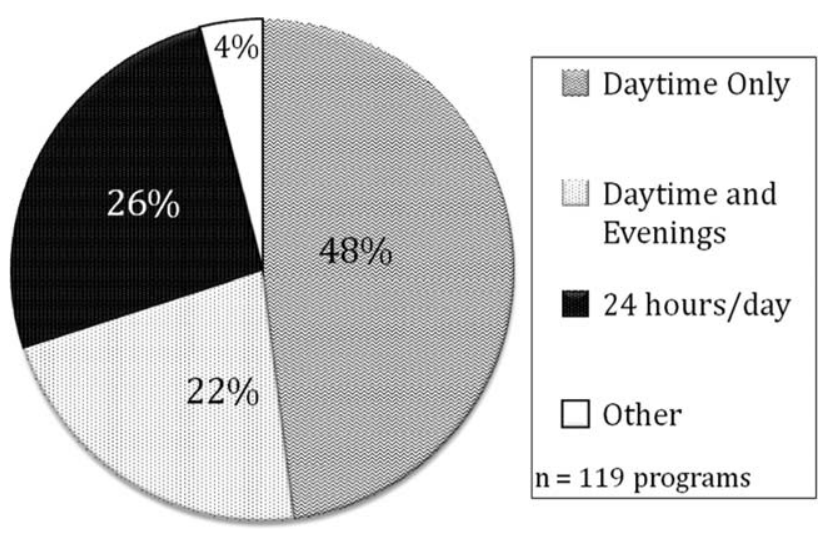

FIG. 4. Of programs with pediatric hospitalists, when are the hospitalist attendings in-house?

seeing select general pediatric admissions and 35\% seeing all general pediatric admissions.

Of the programs without $24 / 7$ pediatric hospital attending coverage, $26 \%$ reported that they are planning to add this coverage within the next 5 years. If this occurred, $41 \%$ of total responding pediatric residency programs would have $24 / 7$ pediatric hospital coverage $(P<0.0001)$ (Figure 5).

\section{DISCUSSION}

Great variation exists in night staffing of pediatric inpatient teaching services. Residency programs have adapted to changes in residency work hours and increased supervision regulations by utilizing night float systems and increasing in-house attending coverage at night. The largest growth of in-house attending physicians at night since these work-hour changes has been pediatric hospitalist attendings. Although hospital medicine has been a rapidly growing field over the past 10 years, many program directors in our study felt that the change in resident work hours was the primary driver of increased in-house attending physicians at night. At the time of this study, pediatric 


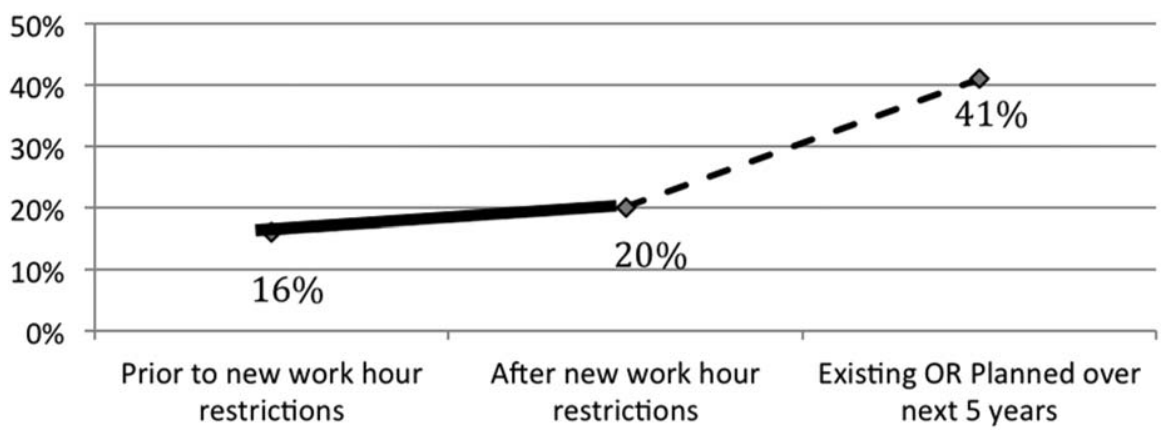

FIG. 5. Pediatric hospitalist attending $24 / 7$ in-house coverage. This in-house coverage may include both supervising and nonsupervising pediatric hospitalist attendings.

program directors are anticipating an even larger increase in this hospitalist coverage over the next 5 years.

\section{Effects of Increased Resident Work-Hour Restrictions on Patient Safety}

The literature is unclear on whether patient safety has improved due to residency work-hour restrictions. Several studies show decreased mortality among highrisk patients, but there are conflicting reports on if patient complications have changed. ${ }^{10-15}$ Two systematic reviews did not show evidence of improved patient safety with increased resident work-hour restrictions. Some of the studies in these reviews showed a change in medical errors, but no increased patient morbidity and mortality. Although residents were less fatigued with new resident work hours, there is also concern that increased resident handoffs, especially with the increase in resident night float, could lead to medical errors. ${ }^{16,17}$

\section{Effects of Increased Resident Work-Hour Restrictions on Resident Education}

There is concern regarding dissatisfaction among residents, nursing, and training physicians with respect to resident education following the change in residency work-hour regulations. A systematic review showed negative perceptions of resident education following resident work-hour restrictions. ${ }^{18}$ Another systematic review assessed all intervention studies that reduced resident work shifts over 16 hours, showing no change in resident education with improved patient safety. ${ }^{19}$ However, multiple studies done following this systematic review show otherwise. ${ }^{14,15,20,21}$ A more recent study showed that although residents were better rested following the shortened work schedule, there was increased work-load intensity while at work, with decreased patient ownership as well as decreased didactic education (a 25\% reduction in ability to attend the noon conference). ${ }^{20}$ This could be related to the increase in resident night float seen in our study, resulting in residents not being present during the daytime when much of the didactic education takes place. The number of patient handoffs dra- matically increased with an association with higher rates of medical errors. ${ }^{14,15,20,21}$ A single-center study looking specifically at resident education before and after resident work-hour restrictions were implemented showed improved resident education. This study is hard to generalize due to increased educational programs and redesign of the inpatient services during this time. ${ }^{22}$ Although the new resident workhour regulations were supposed to increase resident wakefulness, a study on surgical interns found that interns were actually more sleepy on the night float schedule, possibly due to multiple nights of poor sleep without a "post-call day" to make it up. ${ }^{23}$ Our hypothesis on these conflicting studies is that changes in work-hour restrictions could result in improved quality of patient care and improved education with the right mix of increased educational programs (on handoffs) and redesign of inpatient services. It is also worth noting that all studies are biased by the constraint of minimal change in resident workload or residency duration. The basic structure of residency may require change to produce the potentially competing goals of improved patient safety and appropriate medical training.

\section{Effects of Increased Resident Supervision and the Role of the In-House Pediatric Hospitalist at Night}

Although increased resident work hours were an important piece of new 2011 ACGME regulations, they also involved increased resident supervision. This may be more important on nights and weekends where there is increased likelihood of patient morbidity and mortality. ${ }^{24}$ Our study shows an increase of in-house attending coverage at night. Although the reasoning for this is likely multifactorial, a large proportion of program directors attributed it to changes in residency work hours. Our study also showed that many of the programs utilized pediatric hospitalist attendings to increase this coverage. A recent study showed that this increased supervision at night improved both the resident education and perception of patient care. ${ }^{25}$ Despite this, there is still variation in overnight hospitalist attendings' supervision of trainees, even when hospitalist attendings are present. 
Our study showed the majority of hospitalist in-house overnight attendings had roles in teaching and consulting for residents. A different study of internal medicine hospitalist attendings found $61 \%$ of these programs had hospitalist attendings in-house overnight. Only $38 \%$ of these programs had formally defined supervisory roles, with almost $25 \%$ of the programs with overnight coverage involved in nonteaching services only. ${ }^{26}$ although a majority of these hospitalist attending leaders felt formal overnight supervision would improve patient safety and resident education, they were concerned for decreased resident autonomy and increased hospitalist attending workload. ${ }^{26}$

There is little literature specifically on effects of increased resident supervision directly on patient safety since new resident work hours. One large pediatric residency program without 24/7 in-house attending coverage found that decreasing attending presence by phone did not decrease quality of patient care. ${ }^{27}$

Like all studies, our study has limitations that warrant consideration. Program directors were the respondents in our study. Although they were knowledgeable on residency changes, there may be specific questions on attending responsibilities and future direction of pediatric hospitalist services that may be better answered by specific specialty directors. Although our survey asked for information on the responsibilities of pediatric hospitalist attendings at night, we did not specifically examine responsibilities of nonteaching services. We also did not clarify responsibilities of other attendings (PICU, NICU, emergency medicine) and their utilization of nonteaching services. We did have dropout bias toward the end of our survey. We cannot comment on differences between responding and nonresponding programs, although this is minimized by our large response rate, with similar average program size compared to ACGME data. We are limited by a smaller percentage of small programs responding compared to higher response rates of large and medium-sized programs. Although program directors predicted large growth of in-house 24/7 hospitalist coverage, recent economic changes in reimbursement may limit this. ${ }^{28}$ Our initial study prior to implementation of 2011 work hours suggested $31 \%$ of programs planned to add $24 / 7$ coverage within 5 years. ${ }^{9}$ Our current study shows that whereas 24/7 hospitalist coverage is still projected to grow rapidly, it has not yet done so. This could be related to the time to implement 24/7 coverage (hiring staffing for this model and financial concerns) versus the ability of program directors to predict the future of pediatric hospital medicine divisions. Finally, although we feel that the changes in residency work hours likely contributed to the increase in 24/7 hospitalist coverage, this increase is probably multifactorial and could be related to financial and marketing reasons.

\section{CONCLUSIONS}

In conclusion, our study shows that although programs vary in their response to changes in residency work hours, they most commonly utilize night float systems and increased in-house attending coverage at night, especially among pediatric hospitalist attendings. These changes are likely multifactorial, but many programs attribute increased attending in-house nighttime coverage to changes in residency work hours. Pediatric hospitalist attendings have had the largest growth of in-house attending physicians at night, with many programs planning to increase in-house pediatric hospitalist attending coverage at night in the next 5 years. Further investigation is needed to determine the impact of in-house hospitalist attending coverage at night on patient outcomes, supervision, and resident education. In the current economic environment with reimbursement rates and national inpatient volumes continuing to decline, hospitals continue to explore options to lower expenses and boost productivity. ${ }^{28,29}$ The perceived need for 24/7 attending in-house presence may prove to be a financial disincentive for smaller programs and accelerate the shift in pediatric beds to larger, tertiary care settings. A national study may be needed to determine the overall importance and necessity of in-house hospitalist attending coverage at night, with regard to maintaining high levels of patient care and residency education while adapting to new economic constraints.

Disclosures: Dr. Oshimura designed the study, coordinated and supervised the data collection, drafted the initial manuscript, and approved the final manuscript as submitted. She has had full access to all of the data in the study and takes responsibility for the integrity of the data and the accuracy of the data analysis. Dr. Sperring and Dr. Bauer conceptualized and designed the study, reviewed the initial analyses, reviewed and revised the manuscript, and approved the final manuscript as submitted. Dr. Carroll critically reviewed the data collection instruments, analyzed and interpreted the data, reviewed and revised the manuscript, and approved the final manuscript as submitted. Dr. Rauch conceptualized and designed the study, coordinated and supervised data collection, reviewed the initial analysis, reviewed and revised the manuscript, and approved the final manuscript as submitted. There was no funding source for the design and conduct of the study; collection, management, analysis, and interpretation of the data; and preparation, review, or approval of the manuscript; and decision to submit the manuscript for publication. The authors report no conflicts of interest.

\section{References}

1. Samkoff JS, Jacques CH. A review of studies concerning effects of sleep deprivation and fatigue on residents' performance. Acad Med. 1991;66:687-693.

2. Howard SK, Gaba DM, Rosekind MR, Zarcone VP. The risks and implications of excessive daytime sleepiness in resident physicians. Acad Med. 2002;77:1019-1025.

3. Philibert I, Taradejna C. A brief history of duty hours and resident education. https://www.acgme.org/acgmeweb/Portals/0/PDFs/gme11-00-5-11\%5B1\%5D.pdf. Accessed July 26, 2014.

4. Accreditation Council for Graduate Medical Education. Statement of justification/impact for the final approval of common standards related to resident duty hours; September 2002. Available at: www. acgme.org. Accessed November 4, 2013.

5. Gaba DM, Howard SK. Fatigue among clinicians and the safety of patients. N Engl J Med. 2002;347(16):1249-1255.

6. Ulmer C, Wolman DM, Johns MME, eds. Resident Duty Hours: Enhancing Sleep, Supervision, and Safety. Washington, DC: National Academies Press; 2008.

7. Lockley SW, Cronin J, Evans E, et al. Effect of reducing interns' weekly work hours on sleep and attentional failure. N Engl J Med. 2004;351(18):1829-1837. 
8. Accreditation Council for Graduate Medical Education. ACGME approved standards, effective July 2011. Available at: http://www.acgme.org/acgmeweb/Portals/0/PDFs/Common_Program_Requirements_07012011\%5B2\% 5D.pdf. Accessed November 4, 2013.

9. Oshimura J, Sperring J, Bauer B, Rauch D. Inpatient staffing within pediatric residency programs: work hour restrictions and the evolving role of the pediatric hospitalist. J Hosp Med. 2012;7(4):299-303.

10. Philibert I, Nasca T, Brigham T, Shapiro J. Duty-hour limits and patient care and resident outcomes: can high-quality studies offer insight into complex relationships? Ann Rev Med. 2013;64:467-483.

11. Landrigan CP, Rothschild JM, Cronin JW, et al. Effect of reducing interns' work hours on serious medical errors in intensive care units. N Engl J Med. 2004;351:1838-1848.

12. Privette AR, Shackford SR, Osler T, Ratliff J, Sartorelli K, Hebert JC. Implementation of resident work hour restrictions is associated with a reduction in mortality and provider-related complications on the surgical service: a concurrent analysis of 14,610 patients. Ann Surg. 2009;250(2):316-321.

13. Salim A, Teixeira P, Chan L, et al. Impact of the 80-hour workweek on patient care at a level 1 trauma center. Arch Surg. 2007;142(8):708-714.

14. Sen S, Kranzler HR, Didwania AK, et al. Effects of the 2011 duty hour reforms on interns and their patients: a prospective longitudinal cohort study. JAMA Intern Med. 2013;173(8):657-662.

15. Shetty K, Bhattacharya J. Changes in hospital mortality associated with residency work-hour regulations. Ann Intern Med. 2007;147(2):73-80.

16. Fletcher K, Davis S, Underwood W, et al. Systematic review: effects of resident work hours on patient safety. Ann Intern Med. 2004; 141(11):851-857.

17. Peets A, Ayas N. Restricting resident work hours: the good, the bad, and the ugly. Crit Care Med. 2012;40(3):960-966.

18. Mansukhani M, Kolla B, Surani S, et al. Sleep deprivation in resident physicians, work hour limitations, and related outcomes: a systematic review of the literature. Postgrad Med. 2012;124(4):241-249.

19. Levine A, Adusumilli J, Landrigan C. Effects of reducing or eliminating resident work shifts over 16 hours: a systematic review. Sleep. 2010;33(8):1043-1053.
20. Desai SV, Feldman L, Brown L, et al. Effect of the 2011 vs 2003 duty hour regulation-compliant models on sleep duration, trainee education, and continuity of patient care among internal medicine house staff. JAMA Intern Med. 2013;173(8):649-655.

21. Auger KA, Landrigan CP, Gonzalez del Rey JA, Sieplinga KR, Sucharew HJ, Simmons JM. Better rested, but more stressed? Evidence of the effects of resident work hour restrictions. Acad Pediatr. 2012; $12(4): 335-343$.

22. Theobald C, Stover D, Choma N. The effect of reducing maximum shift lengths to 16 hours on internal medicine interns' educational opportunities. Acad Med. 2013;88(4):512-518.

23. Kamine TH, Barron RJ, Lesicka A, Galbraith JD, Millham FH, Larson J. Effects of the new Accreditation Council for Graduate Medical Education work hour rules on surgical interns: a prospective study in a community teaching hospital. Am J Surg. 2012;205(2): 163-168.

24. Nocturnists help avoid night, weekend danger. Healthcare Benchmarks Qual Improv. 2011;18(11):127.

25. Haber L, Lau C, Sharpe B, Arora V, Farnan J, Ranji S. Effects of increased overnight supervision on resident education, decisionmaking, and autonomy. J Hosp Med. 2012;7(8):606-610.

26. Farnan J, Burger A, Boonayasai B, et al. Survey of overnight academic hospitalist supervision of trainees. J Hosp Med. 2012;7: 521-523.

27. Biondi E, Leonard M, Nocera E, Chen R, Arora J, Alverson B. Tempering pediatric hospitalist supervision of residents improves admission process efficiency without decreasing quality of care. J Hosp Med. 2014;9(2):106-110.

28. Albright, E. CMS announces Medicare reimbursement changes for 2014. July 16, 2013. Available at: http://www.insidepatientfinance. $\mathrm{com} /$ revenue-cycle-news/cms-announces-medicare-reimbursementchanges-for-2014. Accessed September 12, 2013.

29. Agency for Healthcare Research and Quality (AHRQ). National estimates on use of hospitals by children from the HCUP Kids Inpatient Database. Available at: http://hcupnet.ahrq.gov/HCUPnet.jsp. Accessed September 12, 2013. 